\title{
Pancreatic ampullary carcinoma with neck metastases: a case
} report

\author{
Murat Aksoy¹, Aziz Sumer*2, Serkan Sari1 ${ }^{1}$ Ozgur Mete ${ }^{3}$, Artur Salmaslioglu ${ }^{4}$ \\ and Yesim Erbil ${ }^{1}$
}

Address: ${ }^{1}$ Department of General Surgery, Faculty of Medicine, Istanbul University, İstanbul, Turkey, ${ }^{2}$ Department of General Surgery, Kas State Hospital, Antalya, Turkey, ${ }^{3}$ Department of Pathology, Faculty of Medicine, Istanbul University, İstanbul, Turkey and ${ }^{4}$ Department of Radiology, Faculty of Medicine, Istanbul University, İstanbul, Turkey

Email: Murat Aksoy - maksoy@hotmail.com; Aziz Sumer* - azizsumer2002@yahoo.com; Serkan Sari - drsekansari@yahoo.com; Ozgur Mete - ozgurmete77@hotmail.com; Artur Salmaslioglu - asalmaslioglu@hotmail.com; Yesim Erbil - yerbil2003@yahoo.com

* Corresponding author

Published: I October 2009

Cases Journal 2009, 2:146 doi:10.1186/1757-1626-2-146

This article is available from: http://www.casesjournal.com/content/2/I//46

(C) 2009 Aksoy et al; licensee BioMed Central Ltd.

This is an Open Access article distributed under the terms of the Creative Commons Attribution License (http://creativecommons.org/licenses/by/2.0), which permits unrestricted use, distribution, and reproduction in any medium, provided the original work is properly cited.
Received: 19 September 2009

Accepted: I October 2009

\begin{abstract}
Background: An 18-year-old Turkish woman was referred with a 6-week history of rapidly enlarging cervical mass at the left side.

Case report: She was diagnosed of ampullary carcinoma for which pancreatoduodenectomy was performed 14 months ago. In our patient with a history of malignancy, a rapidly enlarging neck mass was considered a metastasis to the neck. Tumor resection was performed. Histopathological examination revealed the metastasis of the precedent ampullary adenocarcinoma.
\end{abstract}

Conclusion: Surgery does not improve survival for advanced metastatic ampullary cancer however, it can be mandatory in specific conditions as our patient.

\section{Introduction}

Periampullary neoplasms include carcinomas of the duodenum, ampulla of Vater, distal common bile duct and pancreas. They are considered collectively because of their similar clinical presentation and the difficulty in distinguishing them without examination of a resected specimen $[1,2]$. More than one half of cases have distant metastasis at diagnosis. Most frequent sites of metastasis are local lymph nodes, lung, liver, adrenal glands, kidney and bones [3-7]. To our knowledge, this is the rare report of a ampullary cancer with neck metastasis.

\section{Case presantation}

An 18-year-old Turkish woman was referred from Oncology Department with a 6-week history of rapidly enlarging cervical mass at the left side of her neck which had been detected by the patient herself. She was diagnosed of ampullary carcinoma for which pancreatoduodenectomy was performed 14 months ago. Histopathological examination revealed well-differentiated adenocarcinoma in the ampullary region. Only one lymph node metastasis was present among 35 dissected lymph nodes. Additionaly, peripancreatic fat tissue invasion was also remarked. She has not a family history of pancreas cancer. Gemcitabine was started $1000 \mathrm{mg} / \mathrm{m} 2$ Day 1,8,15/every 28 days for ampullary cancer. After chemotherapy performed, she lost follow-up.

Six months after the chemotherapy, she noticed a rapidly enlarging right neck mass. A $15 \mathrm{~cm}$ soft and painless mass involving the left antero-lateral aspect of the neck was palpable. The mass was apparent by inspection (Figure 1A). 

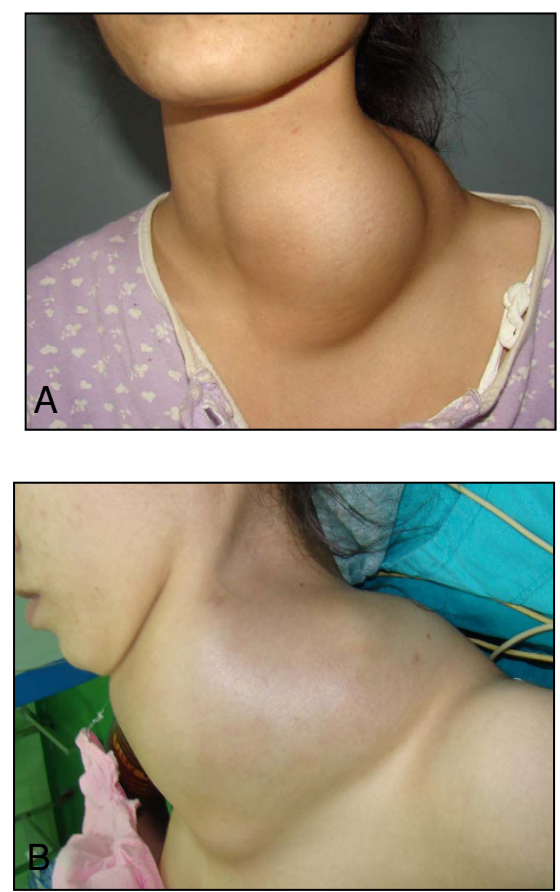

Figure I

(A) Huge neck mass before and (B) after biopsy.
The mass was fixed to the underlying cervical tissues. There was no known past history of thyroid problems and she was euthyroid. Routine blood chemistry including liver function tests and white blood cell count were normal. Her erythrocyte sedimentation rate and CA-19-9 were $55 \mathrm{~mm} / \mathrm{h}$ and $2044 \mathrm{U} / \mathrm{ml}$, respectively. Indirect laryngoscopic evaluation revealed normal vocal cords function.

Ultrasonography revealed a mixed solid- cystic mass, measuring $13 \times 11 \mathrm{~cm}$, with a hyperechogenic capsule. The lesion had a close relationship with the thyroid gland and its borders could not be easily defined. On T2 weighted axial MR images, a hyperintense, dominantly cystic mass with thin borders and papillary vegetations at the posterior wall was occupying the left cervical region. The lesion had displaced the trachea, the thyroid gland and also compressed the left common carotid artery and internal jugular vein. Smaller lesions with similar internal structure could also be detected at the posterosuperior and left posterolateral sides of the primary lesion (Figure 2A). On T1 weighted spin-echo images the main lesion was hyperintense (due to intralesional hemorrhage or presence of proteinaceous material) with isointense papillary components. The smaller lesions were hypointense (Figure 2B). Following contrast material administration, a
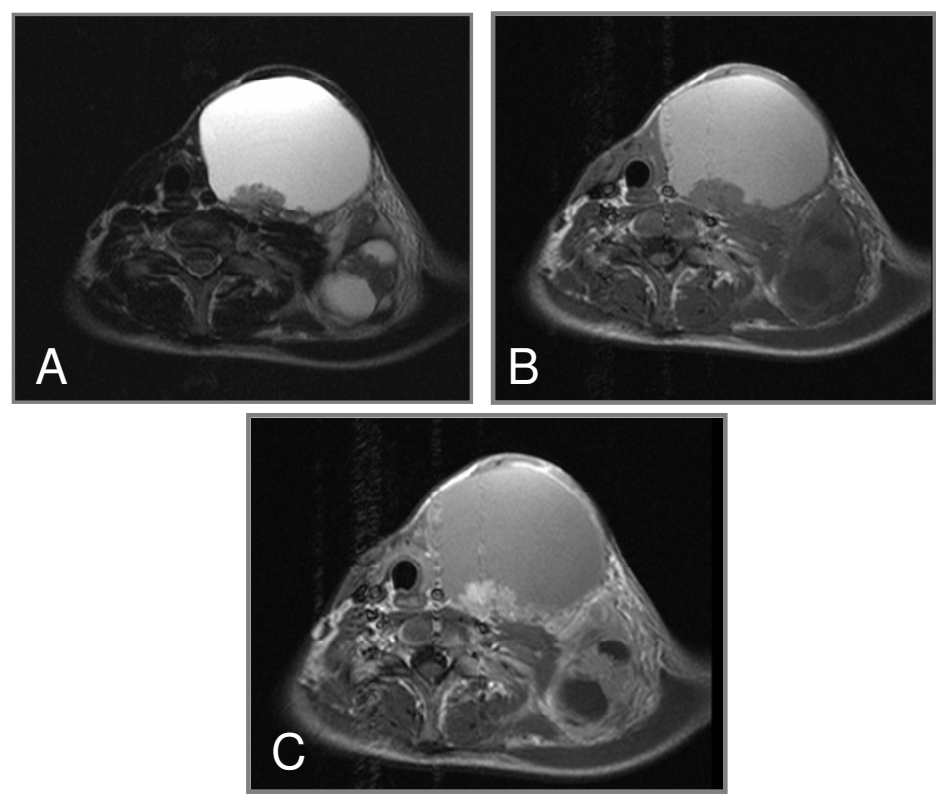

Figure 2

(A) T2 weighted TSE transverse image demonstrates a large, hyperintense cystic mass with polypoid solid components, displacing middle-line structures, including trachea and esophagus, to the right. The left common carotid artery is in contact with the medial wall of the lesion. Smaller lesions with similar internal structure can also be detected at the posterosuperior and left posterolateral sides. (B). TI weighted SE transverse image demonstrates spontaneous hyperintensity in the primary lesion, consistent with intralesional hemorrhage or proteinaceous material. Papillary vegetations are isointense. The smaller posterior components are hypointense with isointense solid vegetations. (C). Contrast enhanced TI weighted SE transverse image demonstrates heterogeneous enhancement at the papillary components. 

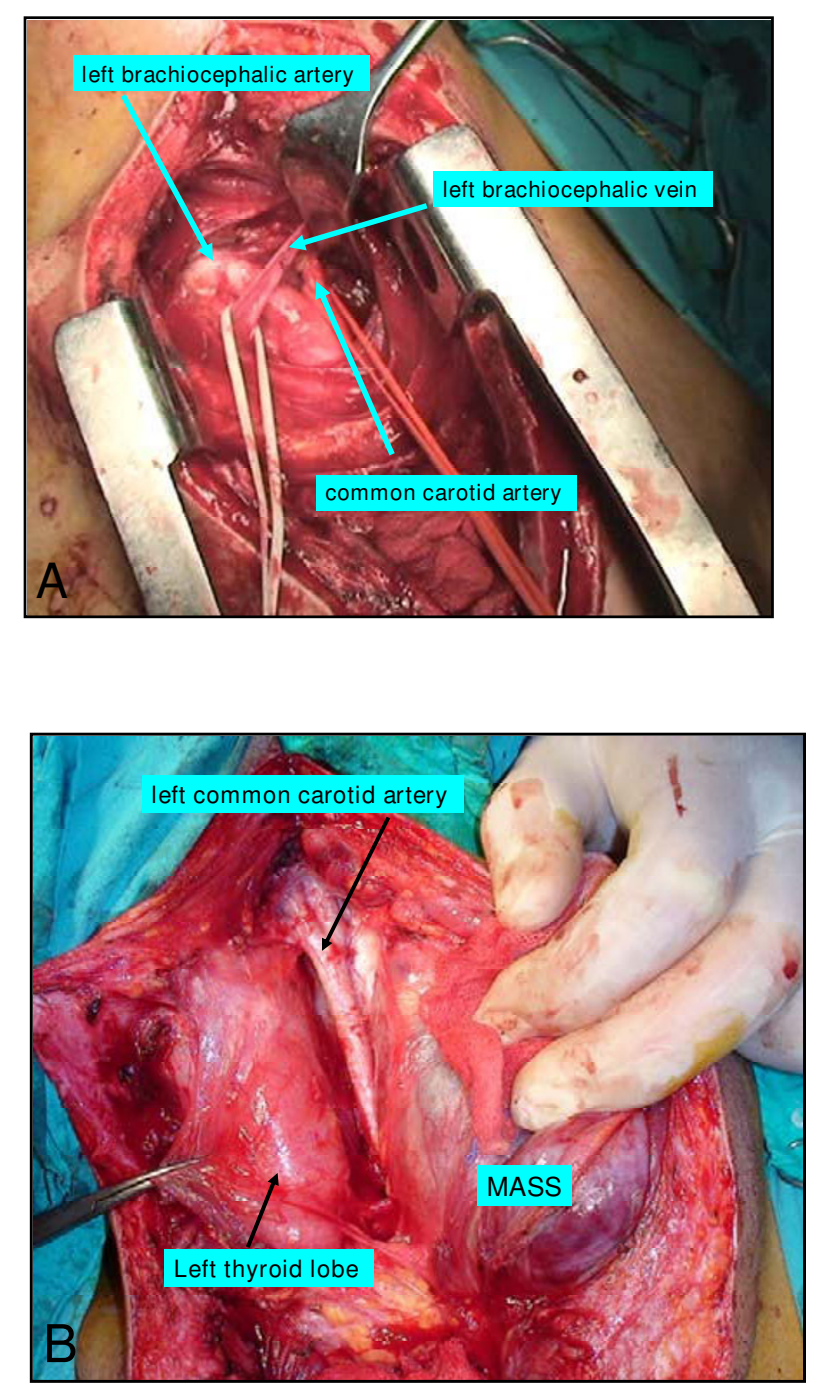

Figure 3

(A) Isolated left brachiocephalic artery and vein, and left common carotid artery; (B) The appearance of carotid artery, internal jugular vein, left thyroid lobe and cervical mass.

heterogeneous enhancement could be noted at the papillary components (Figure 2C).

In our patient with a history of malignancy, a rapidly enlarging neck mass was considered a metastasis to the neck. Since imaging studies suggested malignant tumor, a Tru-cut biopsy was performed. Cytologic findings did not provide any further information. After Tru-cut biopsy, severe compressive symptoms and stridor were occured. Those symptoms, accompanied by the enlargement of the mass, were suggestive of intralesional bleeding following biopsy (Figure 1B).

\section{Surgical technique}

The patient underwent sternotomy and the left brachiocephalic artery and vein, and left common carotid artery, and left subclavian artery were isolated for vascular control. At the neck exploration, the left cervical mass was voluminous, and adherent to surrounding structures including the carotid artery, internal jugular vein, and left vagal nerve. Left thyroid lobe was found normal in appearance. Left common carotid artery and vagal nerve were liberated from the tumor. Because the tumor invaded left internal jugular vein, it was ligated and divided. After mobilization of the tumor from the adjacent structures, tumor resection was completed (Figure 3A, B). She had an uneventful postoperative course and was discharged home on postoperative day 3 . The histopathological examination revealed a metastatic adenocarcinoma containing areas of extensive lymphovascular tumor thrombus (Figure 4A, B). Sections were free of any lymphoid parenchyma and for this reason the mass is regarded as being a metastatic soft tissue mass in the neck region. Anti-cytokeratin 7 and CEA (Carcinoembryogenic antigen) were diffuse and strongly positive in the tumor cells, whereas anti-TTF1 and cytokeratin 20 were negative (Figure 4C, D). Antigenic and morphological features lead us to diagnose the mass as the metastasis of the precedent periampullary adenocarcinoma. At a follow-up visit 3 months after surgery, she remained symptom-free, and a repeat US showed no evidence of recurrence.

\section{Discussion}

Overall, periampullary cancers account for $5 \%$ of all gastrointestinal tract malignancies, which can be divided into four groups of tumor entities: periampullary adenocarcinoma originates from the pancreatic duct (pancreatic carcinoma), the mucosa of the ampulla of Vater (ampullary carcinoma), the common distal bile duct segment (distal cholangiocellular carcinoma) or the duodenum (duodenal adenocarcinoma) $[1,2]$.

Panreatic cancer occurs most frequently among the periampullary cancers, accounting for 3\% of all gastrointestinal cancers. Carcinoma of the ampulla of Vateri is the second most common periampullary malignancy. Less frequent are distal cholangiocellular carcinoma and duodenal adenocarcinoma of the periampullary region (1). The prognosis of a 5 -year survival rate between $1 \%$ and $10 \%$ for all periampullary cancers still remains a frustrating challenge. However, it has been shown that periampullary tumors, not arising from the pancreatic duct, have a much beter outcome [3].

Morbidity and mortality from pancreatic cancer is conspicuously associated with metastasis; the most frequent sites of metastasis are local lymph nodes, lung, liver, adrenal glands, kidney and bones. Patients with pancreatic 

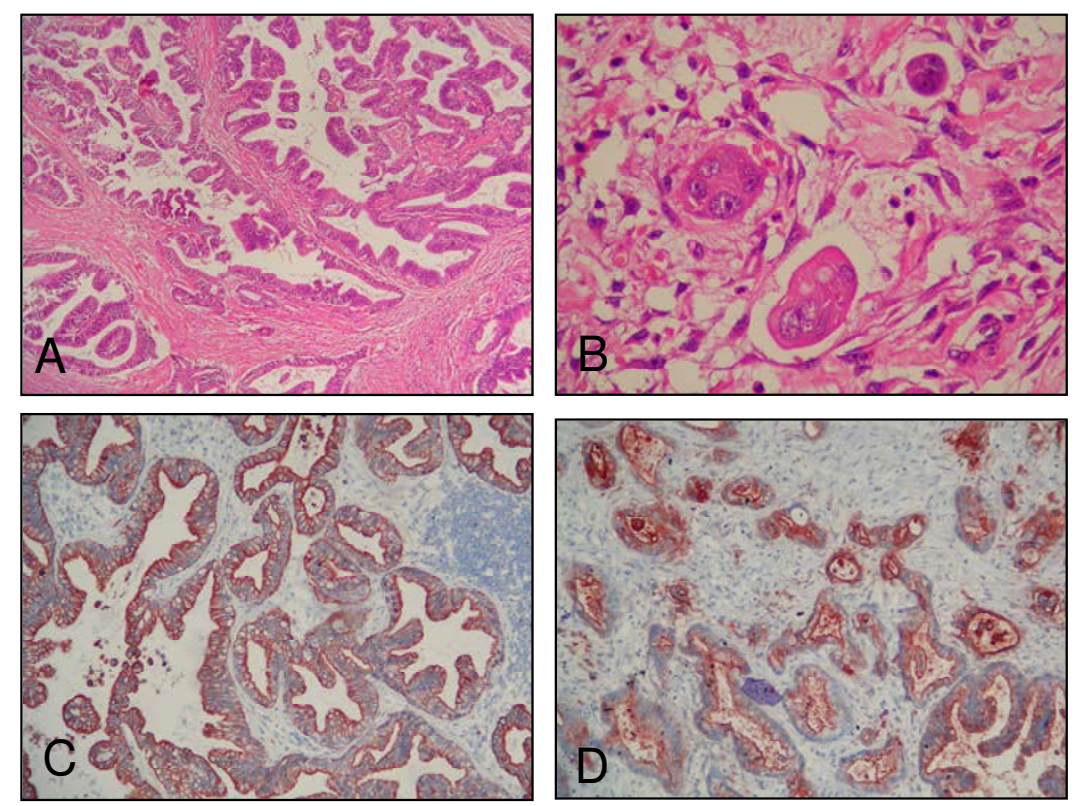

\section{Figure 4}

(A) Metastatic adenocarcinoma forming a mass in the neck soft tissue (H\&E, ×20); (B) Lymphovascular tumor thrombus in the surrounding soft tissue (H\&E, ×40); (C) Anti-cytokeratin 7 positivity in the tumor cells (Anticytokeratin 7, ×20); (D) Anti-CEA positivity in the tumor cells (Anti-CEA, ×40).

cancer have only very rarely been reported to develop metastatic lesions to the brain, skin, and larynx (3-7). Cervical lymph node and brain metastases from pancreatic cancer have rarely been seen and most of them were identified in advanced stage $[5,8]$. In our case, histopathologic examination did not reveal any lymphoid tissue, which suggests a soft tissue metastasis to the neck. However, it is not possible to prove this hypothesis. Despite the fact that our patient was in an advanced stage, the excision was performed to prevent respiratory difficulties.

While surgery does not improve survival for advanced metastatic ampullary cancer, it can be mandatory in specific conditions as our patient.

\section{Consent}

Written informed consent was obtained from the patient for publication of this case report and accompanying images. A copy of the written consent is available for review by the Editor-in-Chief of this journal.

\section{Competing interests}

The authors declare that they have no competing interests.

\section{Authors' contributions}

MA and YE carried out the operation, AS and YE were contributor in writing the manuscript. SS participated in discussing of the manuscript. OM performed the histological examination of the mass. ARS performed the MRI image examination of the mass. All authors read and approved the final manuscript.

\section{References}

I. Berberat PO, Künzli BM, Gulbinas A, Ramanauskas T, Kleeff J, Müller MW, Wagner M, Friess H, Büchler MW: An audit of outcomes of a series of periampullary carcinomas. Eur J Surg Oncol 2009, 35: $187-9 \mid$.

2. Diener MK, Knaebel HP, Heukaufer C, Antes G, Büchler MW, Seiler $C M$ : A systematic review and meta-analysis of pylorus-preserving versus classical pancreaticoduodenectomy for surgical treatment of periampullary and pancreatic carcinoma. Ann Surg 2007, 245: 187-200.

3. Edwards BK, Howe HL, Ries LA, Thun MJ, Rosenberg HM, Yancik R, Wingo PA, Jemal A, Feigal EG: Annual report to the nation on the status of cancer, 1973-1999, featuring implications of age and aging on U.S. Cancer 2002, 94:2766-92.

4. Yendluri V, Centeno B, Springett GM: Pancreatic cancer presenting as a Sister Mary Joseph's nodule: case report and update of the literature. Pancreas 2007, 34:I6I-4.

5. Caricato M, Borzomati D, Ausania F, Garberini A, Rabitti C, Tonini G, Coppola R: Cerebellar metastasis from pancreatic adenocarcinoma. A case report. Pancreatology 2006, 6:306-8.

6. Hafez H: Cutaneous pancreatic metastasis: A case report and review of literature. Indian J Cancer 2007, 44: I I -4.

7. Oku T, Hasegawa M, Watanabe I, Nasu M, Aoki N: Pancreatic cancer with metastasis to the larynx. J Laryngol Otol 1980, 94:1205-9.

8. Funakoshi A, Senju T, Sumii T: Two cases of advanced pancreatic cancer with cervical lymph node or liver metastasis responding well to S-I monotherapy. Gan To Kagaku Ryoho 2006, 33:1505-9. 